\title{
La modalidad mixta una alternativa de continuidad de estudios para egresados inmersos en el campo laboral
}

\section{Blended learning an alternative to continue higher education studies for Associate's degree graduates immersed in the labor market}

\author{
DOMINGUEZ-GUTU, Jesus $\dagger^{*}$, GORDILLO-ESPINOZA, Emmanuel y CONSTANTINO- \\ GONZÁLEZ, Fernando Exiquio
}

Universidad Tecnológica de la Selva

ID $1^{\text {er }}$ Autor: Jesús, Dominguez-Gutu / ORC ID: 0000-0001-8025-6089, CVU CONACYT ID: 524210

ID $1^{\text {er }}$ Coautor: Emmanuel, Gordillo-Espinoza / ORC ID: 0000-0002-2467-8209, CVU CONACYT ID: 657274

ID $2^{\text {do }}$ Coautor: Fernando Exiquio, Constantino-González / ORC ID: 0000-0002-9701-1990, CVU CONACYT ID: 79617

\begin{abstract}
Resumen
La modalidad mixta (Blended Learning) busca implementar el uso de las Tecnologías de la Información y Comunicación (TIC's) para brindar estudios de Educación Superior Tecnológica a personas que se encuentran inmersas en el mercado laboral. Esta modalidad fue implementada por primera ocasión en la Universidad Tecnológica de la Selva, ubicada en la ciudad de Ocosingo, Chiapas, para el programa educativo de Ingeniería en Tecnologías de Información. Esta investigación se centró en conocer la percepción de los estudiantes que cursaron su carrera profesional bajo esta modalidad y que actualmente se encuentran empleados, el estudio se realizó bajo un enfoque cuantitativo no exprimental con diseño transaccional descriptivo, que evaluó la plataforma educativa utilizada, a los docentes y a la modalidad de estudios, se utilizó como herramienta de recolección de información una encuesta con preguntas cerradas bajo la escala de Likert y una pregunta abierta para sugerencias que permitían mejorar a esta modalidad. Los resultados demuestran que para los estudiantes que se encuentran inmersos en el campo laboral, la modalidad mixta es excelente para continuar con sus estudios profesionales, ya que pueden administrar su tiempo, además esperan mejorar su desempeño profesional, así como, sus condiciones económicas al conseguir un título profesional de ingeniería.
\end{abstract}

Modalidad mixta, Plataforma educativa, Recursos didácticos

\begin{abstract}
The mixed methodology (Blended Learning) seeks to implement the use of Information and Communication Technologies (ICT) to provide studies of Technological Higher Education to people who are immersed in the labor market. This modality was implemented for the first time at the Universidad Tecnologica La Selva, located in the city of Ocosingo, Chiapas, for the educational program of Information Technology Engineering. This research focused on knowing the perception of the students who pursued their professional career under this modality and who are currently employed, the study was carried out under a non-experimental quantitative approach with descriptive transactional design, which evaluated the educational platform used, the teachers and the study modality. A survey with closed questions under the Likert scale and an open question for suggestions that allowed improving this modality were used as an information collection tool. The results show that for students who are immersed in the labor field, blended learning is excellent to continue with their professional studies since they can manage their time, moreover, they hope to improve their professional performance as well as their economic conditions when getting a professional engineering degree.
\end{abstract}

Mixed mode (blended learning), Educational platform, Didactic resources

Citación: DOMINGUEZ-GUTU, Jesús, GORDILLO-ESPINOZA, Emmanuel y CONSTANTINO-GONZÁLEZ, Fernando Exiquio. La modalidad mixta una alternativa de continuidad de estudios para egresados inmersos en el campo laboral. Revista de Educación Superior. 2019 3-10: 12-20

\footnotetext{
* Correspondencia del Autor (Correo electrónico: jdominguez@laselva.edu.mx)

$\dagger$ Investigador contribuyendo como primer autor.
} 


\section{Introducción}

En los últimos años, el avance de las Tecnologías de la Información y la Comunicación han permeado los múltiples escenarios y formas de abordar los procesos de enseñanza - aprendizaje en la educación superior.

En la actualidad, no existe impedimento de las distancias para la educación, ya no es necesario asistir a salones, sino que desde el lugar donde nos encontremos podemos acceder a estudios de formación académica por medio de la educación virtual. (Sierra, 2011).

La modalidad mixta (Blended Learning), trata de un modelo hibrido a través del cual, los tutores pueden hacer uso de sus metodologías de aula para una sesión presencial y al mismo tiempo potenciar el desarrollo de las temáticas, a través de una plataforma virtual. (Vera, 2008).

De acuerdo con Mortis, et al. (2015), la calidad de los programas educativos a distancia con apoyo de la tecnología, deben cumplir con los siguientes requerimientos: a) contenidos de calidad, b) tutoría integral, c) comunicación multidireccional con enfoque colaborativo, d) estructura organizativa y de gestión adecuada para la virtualidad y e) plataforma tecnológica o entorno virtual adecuado.

En una modalidad mixta, se diseñan sesiones presenciales que ayudan a los estudiantes a aclarar dudas y verificar progresos en la construcción de los resultados de aprendizaje, a través de exposiciones del profesor y estudiantes, discusiones de grupos, realización de prácticas, aplicación de instrumentos de evaluación, entre otros; por otro lado, las sesiones virtuales representan la parte de la dedicación del estudiante para su aprendizaje, la comunicación con el docente se canaliza a través de la plataforma virtual, las actividades que se incluyen son la investigación bibliográfica, lecturas de apoyo, discusiones en foros, trabajo colaborativo entre estudiantes, estudio de casos, entre otros; aunado a que el estudiante debe construir su propio conocimiento, a través de la búsqueda, selección y procesamiento de la información (Fernández y Panadeiro, 2009).

\section{Marco Teórico}

\section{Blended Learning (b-learning)}

La definición más precisa de b-learning se describe como aquel modo de aprender que combina la enseñanza presencial y la virtual (Coaten, 2003), o la combinación de tecnología e instrucción tradicional en el aula (Marsh, et al., 2003).

Por lo tanto, el b-learning se aproxima más a un modelo de formación híbrido que combina lo mejor del aprendizaje presencial con funcionalidades del aprendizaje electrónico para potenciar las fortalezas y disminuir las debilidades de ambas modalidades (Contreras, et al., 2011).

En esta modalidad, el docente se desenvuelve como formador on-line (a distancia) y como educador en las aulas de aprendizaje (cursos presenciales), y la combinación de éstas estará diseñada en las necesidades específicas de cada asignatura.

De acuerdo con Almarza y Pirela (2010), los docentes deben construir materiales digitales que apoyen al proceso de enseñanza - apredizaje virtual, integrando la tecnología, conectividad, contenidos y recursos humanos, en donde los estudiantes puedan buscar, manipular y colaborar con los contenidos, a través de las tecnologías disponibles.

\section{B-Learning en México}

La evolución del b-learning en latinoamérica se debió a la importancia que cada país le proporcionó a este tipo de educación y a las políticas públicas implementadas para poder ofertar educación a distancia, entre los países se destacan Colombia, Panamá, Brasil, Ecuador, Chile, Perú, Puerto Rico y México (Fernández y Vallejo, 2014).

El modelo b-learning se ha consolidado en latinoamerica, como una opción de educación que mezcla el aprendizaje, a través del uso de tecnologías, entre ellas, plataformas virtuales y formas de comunicación síncrona y asíncrona; con el aprendizaje presencial en el aula de clases. (Zubieta y Rama, 2015). 
En México, los pioneros en incluir la educación virtual son el Instituto Tecnológico de Estudios Superiores de Monterrey (ITESM) y la Universidad Autónoma de México (UNAM) quiénes incursionaron en este tipo de educación desde los años 80's. En el año 2012, se crea la Universidad Abierta y a Distancia de México (UNaDM) como una institución esencialmente en línea (Fernández y Vallejo, 2014). En la actualidad, existen diversas Instituciones de Educación Superior públicas y privadas que han iniciado con la oferta de programas de estudio bajo las diferentes modalidades virtuales. En el Subsistema de las Universidades Tecnológicas y Politécnicas, son 4 universidades que ofertan programas de estudios bajo una modalidad mixta, siendo una enorme área de oportunidad para incrementar la cobertura de dichos programas que se ofertan en las universidades de este subsistema.

En el mundo actual la tecnología se ha incorporado a la vida cotidiana. El alumno ahora utiliza diferentes dispositivos para buscar información y navegar, por lo que éstas personas son consideradas nativos digitales (Chávez, 2015). De acuerdo a una encuesta relizada a los prospectos a cursar la modalidad mixta en la Universidad Tecnológica de la Selva, indica que el $94.8 \%$ tiene acceso a Internet, mediante una computadora o dispositivo móvil. Por otra parte, la educación ha empezado a evolucionar de un lugar físico llamado salón de clases a un lugar virtual que simula un aula de clases. Este espacio es conseguido con los sistemas de gestión del aprendizaje (LMS). La incorporación de estas plataformas educativas ha llevado a la educación a un aprendizaje electrónico (e-learning) o un aprendizaje combinado (b-learning). (Montemayor, 2015)

Uno de los objetivos del Plan de Desarrollo Institucional 2013 - 2018 de la Universidad fue "elevar la calidad de la educación en el estado", implementando la estrategia de "impulsar otras modalidades educativas en el estado", por lo que, en el 2017 se implementa por primera ocasión la modalidad mixta, ofertándose el programa educativo de la Ingeniería en Tecnologías de la Información, siendo la única universidad pública de la región selva del estado de Chiapas en ofertar ésta modalidad; en ese año, se inscribieron 24 estudiantes egresados de Técnico Superior Universitario inmersos en el campo laboral, haciendo uso de la plataforma educativa Moodle.
El presente estudio evaluó las percepciones de los estudiantes que actualmente se encuentran laborando y que cursaron la modalidad mixta en la universidad, con el fin de considerar sus opiniones para mejorar la plataforma educativa utilizada, el desempeño docente en esta modalidad y aspectos que generen conocimientos en la toma de decisiones para la parte directiva de la institución, que permita mejorar y consolidar a esta modalidad.

\section{Objetivo}

El presente estudio de investigación tuvo como objetivo medir la perspectiva de los estudiantes al cursar sus estudios a través de una modalidad mixta.

\section{Metodología}

De acuerdo con Hernández, Fernández y Baptista (2014), se empleó un enfoque cuantitativo no experimental descriptivo que permitió medir la percepción de los estudiantes inmersos en el campo laboral, que estudiaron su carrera profesional en una modalidad mixta, evaluando la plataforma educativa utilizada, a sus docentes y a la modalidad de estudios. La muestra del estudio utilizada fue no probabilística e intencional, que constó de un grupo de 15 estudiantes inscritos en el programa académico de la Ingeniería en Tecnologías de la Información bajo la modalidad mixta, segmentado por género en 12 hombres y 3 mujeres, todos ellos inmersos en el campo laboral y con un estado civil de casado. Una técnica de recolección de datos como la encuesta, tiene como "finalidad la de obtener de manera sistemática medidas sobre los conceptos que se derivan de una problemática de investigación previamente construida" (LópezRoldan y Fachelli, 2015, p. 8). Por lo que, el instrumento para la recolección de datos utilizado fue una encuesta en línea, a través de GoogleForms, con preguntas cerradas bajo la escala de Likert, además una pregunta abierta para sugerencias que permitían mejorar la modalidad mixta. Las preguntas de la encuesta midieron 3 variables: plataforma educativa con 5 preguntas con opciones de Totalmente de acuerdo, De acuerdo, Indiferente, En desacuerdo, Totalmente en desacuerdo, docentes con 7 preguntas y modalidad de estudios con 8, ambas con opciones de Excelente, Muy bien, Bien, Regular y Malo, en los tres casos se incluyó una pregunta abierta para sugerencias. 
El instrumento se aplicó a 15 estudiantes en el mes de diciembre del 2018, cuando cursaban el décimo cuatrimestre de su carrera profesional, previo al cuatrimestre de su estadía en el sector productivo.

\section{Resultados}

Con el propósito de mantener el anonimato en la exposición de los resultados, se utilizarán abreviaturas para referirnos a las opiniones de los estudiantes (OpE\#), donde $\mathbf{O p}$ se refiere a una opinión, $\mathbf{E}$ se refiere a estudiante y \# se enumeró al estudiante que realizó una opinión, de acuerdo al momento en que ingresaron las respuestas a la encuesta.

\section{Plataforma educativa}

En la Tabla 1, se puede observar los resultados sobre la opinión de los estudiantes con respecto a la "plataforma educativa" que utilizaron para su formación profesional.

TD $=$ Totalmente de acuerdo, $\mathbf{D}=$ De acuerdo, $\mathbf{I}$ $=$ Indiferente, $\mathbf{E D}=$ En desacuerdo, TED = Totalmente en desacuerdo

\begin{tabular}{|c|c|c|c|c|c|}
\hline Ítem & TD & D & $\mathbf{I}$ & ED & TED \\
\hline $\begin{array}{l}\text { Imagen } \\
\text { institucional }\end{array}$ & $\begin{array}{l}7 \\
46.67 \%\end{array}$ & $\begin{array}{l}7 \\
46.67 \%\end{array}$ & $\begin{array}{l}0 \\
0 \%\end{array}$ & $\begin{array}{l}1 \\
6.67 \%\end{array}$ & $\begin{array}{l}0 \\
0 \%\end{array}$ \\
\hline $\begin{array}{lr}\text { Presentación } & \text { de } \\
\text { recursos } & \text { y } \\
\text { actividades } & \end{array}$ & $\begin{array}{l}5 \\
33.33 \%\end{array}$ & $\begin{array}{l}8 \\
53.33 \%\end{array}$ & $\begin{array}{l}0 \\
0 \%\end{array}$ & $\begin{array}{l}2 \\
13.33 \%\end{array}$ & $\begin{array}{l}0 \\
0 \%\end{array}$ \\
\hline $\begin{array}{ll}\text { Facilidad } & \text { de } \\
\text { localización } & \text { de } \\
\text { los recursos } & \end{array}$ & $\begin{array}{l}6 \\
40 \%\end{array}$ & $\begin{array}{l}6 \\
40 \%\end{array}$ & $\begin{array}{l}0 \\
0 \%\end{array}$ & $\begin{array}{l}3 \\
20 \%\end{array}$ & $\begin{array}{l}0 \\
0 \%\end{array}$ \\
\hline $\begin{array}{l}\text { Facilidad de } \\
\text { localización de } \\
\text { las actividades } \\
\text { evaluativas }\end{array}$ & $\begin{array}{l}6 \\
40 \%\end{array}$ & $\begin{array}{l}7 \\
46.67 \%\end{array}$ & $\begin{array}{l}0 \\
0 \%\end{array}$ & $\begin{array}{l}2 \\
13.33 \%\end{array}$ & $\begin{array}{l}0 \\
0 \%\end{array}$ \\
\hline Disponibilidad & $\begin{array}{l}4 \\
26.67 \%\end{array}$ & $\begin{array}{l}10 \\
66.67 \%\end{array}$ & $\begin{array}{l}0 \\
0 \%\end{array}$ & $\begin{array}{l}1 \\
6.67 \%\end{array}$ & $\begin{array}{l}0 \\
0 \%\end{array}$ \\
\hline
\end{tabular}

Tabla 1 Estudio de estudiantes sobre la plataforma educativa utillizada

Los resultados obtenidos con relación a la presentación de recursos y actividades y su facilidad de localización, coincide con los resultados de Alonso (2010), donde los estudiantes evalúan la "Idoneidad sitio web (navegación sencilla de la plataforma y funcionalidades fáciles de comprender) por encima de la media establecida" (p. 8), además con relación a la disponibilidad de la plataforma, en el mismo estudio, los estudiantes evalúan el "Funcionamiento de la plataforma por encima de la media establecida" (p. 8), sin embargo, comenta que "es la segunda dimensión de su estudio con el valor medio más bajo" (p. 8).
Sobre este estudio, los estudiantes mencionan algunas sugerencias:

"El diseño de la plataforma, se puede mejorar, así como el uso de materiales didácticos, la plataforma tuvo un diseño estándar el cual no presentaba algo institucional" (OpE5).

"Algunos profesores colocaban sus recursos en diferentes ubicaciones de la plataforma y no en la página principal, por lo que dificulta su ubicación o simplemente se pasaba por alto esa actividad o recurso." (OpE10).

\section{Docentes}

En la Tabla 2, se puede observar los resultados sobre la opinión de los estudiantes con respecto a sus "docentes" que les impartieron asignaturas bajo la modalidad mixta.

$\mathbf{E}=$ Excelente, $\mathbf{M B}=$ Muy bien, $\mathbf{B}=$ Bien, $\mathbf{R}=$ Regular, $\mathbf{M}=$ Malo

\begin{tabular}{|c|c|c|c|c|c|}
\hline Ítem & $\mathbf{E}$ & MB & B & $\mathbf{R}$ & $\mathbf{M}$ \\
\hline $\begin{array}{l}\text { Recursos } \\
\text { didácticos } \\
\text { proporcionados }\end{array}$ & $\begin{array}{c}1 \\
6.67 \%\end{array}$ & $\begin{array}{c}7 \\
46.67 \%\end{array}$ & $\begin{array}{c}4 \\
26.67 \%\end{array}$ & $\begin{array}{c}3 \\
20 \%\end{array}$ & $\begin{array}{c}0 \\
0 \%\end{array}$ \\
\hline $\begin{array}{l}\text { Recursos } \\
\text { proporcionados } \\
\text { fueron } \\
\text { suficientes para } \\
\text { adquirir tus } \\
\text { conocimientos }\end{array}$ & $\begin{array}{c}1 \\
6.67 \%\end{array}$ & $\begin{array}{c}2 \\
13.33 \%\end{array}$ & $\begin{array}{c}8 \\
53.33 \%\end{array}$ & $\begin{array}{c}3 \\
20 \%\end{array}$ & $\begin{array}{c}1 \\
6.67 \%\end{array}$ \\
\hline $\begin{array}{l}\text { Actividades } \\
\text { evaluativas } \\
\text { relacionadas } \\
\text { con los recursos } \\
\text { proporcionados }\end{array}$ & $\begin{array}{c}1 \\
6.67 \%\end{array}$ & $\begin{array}{c}7 \\
46.67 \%\end{array}$ & $\begin{array}{c}5 \\
33.33 \%\end{array}$ & $\begin{array}{c}2 \\
13.33 \%\end{array}$ & $\begin{array}{c}0 \\
0 \%\end{array}$ \\
\hline $\begin{array}{l}\text { Atención de } \\
\text { dudas en las } \\
\text { sesiones Online }\end{array}$ & $\begin{array}{c}4 \\
26.67 \%\end{array}$ & $\begin{array}{c}9 \\
60 \%\end{array}$ & $\begin{array}{c}2 \\
13.33 \%\end{array}$ & $\begin{array}{c}0 \\
0 \%\end{array}$ & $\begin{array}{c}0 \\
0 \%\end{array}$ \\
\hline $\begin{array}{lr}\text { Calificación } & \text { de } \\
\text { actividades, } & \text { de } \\
\text { acuerdo } & \mathrm{a} \\
\text { criterios } & \mathrm{de} \\
\text { evaluación } & \\
\end{array}$ & $\begin{array}{c}1 \\
6.67 \%\end{array}$ & $\begin{array}{c}6 \\
40 \%\end{array}$ & $\begin{array}{c}6 \\
40 \%\end{array}$ & $\begin{array}{c}2 \\
13.33 \%\end{array}$ & $\begin{array}{c}0 \\
0 \%\end{array}$ \\
\hline \begin{tabular}{lr}
\multicolumn{2}{l}{ Retroalimentaci } \\
ón & de \\
actividades, & de \\
acuerdo & a \\
criterios & de \\
evaluación &
\end{tabular} & $\begin{array}{c}2 \\
13.33 \\
\%\end{array}$ & $\begin{array}{c}6 \\
40 \%\end{array}$ & $\begin{array}{c}7 \\
46.67 \\
\%\end{array}$ & $\begin{array}{c}0 \\
0 \%\end{array}$ & $\begin{array}{c}0 \\
0 \%\end{array}$ \\
\hline $\begin{array}{l}\text { Dominio de las } \\
\text { asignaturas } \\
\text { impartidas }\end{array}$ & $\begin{array}{c}2 \\
13.33 \\
\% \\
\end{array}$ & $\begin{array}{c}7 \\
46.67 \\
\% \\
\end{array}$ & $\begin{array}{c}6 \\
40 \%\end{array}$ & $\begin{array}{c}0 \\
0 \%\end{array}$ & $\begin{array}{c}0 \\
0 \%\end{array}$ \\
\hline
\end{tabular}

Tabla 2 Estudio de estudiantes sobre los docentes que les impartieron asignaturas en la modalidad mixta Fuente: Datos de la investigación, 2019 
Los resultados obtenidos con relación a la atención de dudas en las sesiones online y retroalimentación de actividades de acuerdo a criterios de evaluación, coincide con el estudio realizado por Mortis, et al. (2015), en donde los estudiantes mencionan que "la retroalimentación se debe de dar de manera rápida y oportuna para que no haya fallas en la constestación de las dudas" (p. 88), asimismo, la Asociación de Internet MX en el estudio de Educación en Línea 2018, menciona que una de las 3 mayores expectativas de los estudiantes que cursan una modalidad mixta es "contar con evaluación y retroalimentación continua" (p. 11), en el estudio efectuado por Alonso (2010), presenta un resultado muy satisfactorio en el "Tiempo de respuesta del profesor-tutor para la atención de las dudas y recomendaciones sobre los trabajos y la calidad de los mismos" (p. 6).

Por otro lado, la Asociación de Internet MX en el estudio del 2017, en sus resultados menciona que la mayor expectativa de los estudiantes que cursan una modalidad mixta es "contar con profesores de calidad" (p. 27), también Alonso (2010), menciona que "uno de los indicadores con mejor valoración por parte de los alumnos fue el Dominio de la materia por parte del profesor-tutor" (p. 6), coincidiendo con los resultados obtenidos en el domino de las asignaturas impartidas por los docentes.

Mortis, et al. (2015), menciona que "es un reto para los profesores cuando son ellos mismos quienes tienen que desarrollar los materiales didácticos digitales, debido al tiempo y al esfuerzo que deben invertir para prepararlos (...)" (p. 77), por ello, los estudiantes resaltan algunas sugerencias como:

"Que los docentes elaboren su material para impartir clases, ya que se ha visto que algunos de ellos únicamente descargan material de la red" (OpE1).

"Que algunos docentes aporten más videos relacionados a las actividades y sus materias" (OpE11).

Además, sugieren:

"Proporcionar capacitación a los
docentes sobre, diferentes técnicas de
aprendizaje online." (OpE3).

"Proporcionar capacitación a los aprendizaje online." (OpE3).
"Hay profesores que se dedican a dar bien sus clases y hay docentes que no saben ni lo que piden" (OpE7).

\section{Modalidad de estudios}

En el gráfico 1, se presentan las opiniones de los estudiantes de acuerdo al tiempo que le dedicaron a sus estudios, existiedo una opinión favorable hacia la modalidad de estudios.

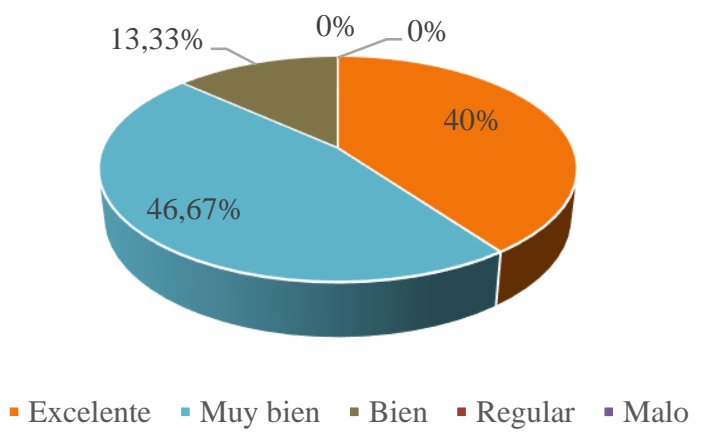

Gráfico 1 Opinión hacia la modalidad, con respecto al tiempo de dedicación a sus estudios

Fuente: Datos de la investigación, 2019

Todos los estudiantes se encuentran inmersos en el campo laboral, el resultado de sus opiniones fue muy favorable hacia la modalidad de estudios (ver gráfico 2).

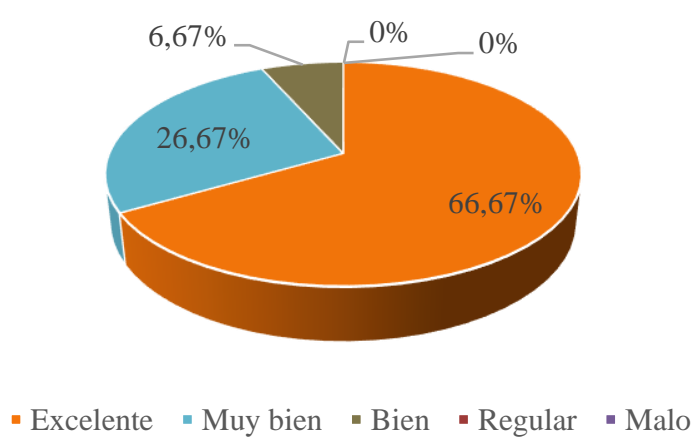

Gráfico 2 Opinión hacia la modalidad, con respecto a las actividades académicas desarrolladas.

Fuente: Datos de la investigación, 2019

Conocer la opinión de los estudiantes con respecto a las actividades académicas que desarrollaban cada semana fue muy interesante, toda vez que el resultado fue muy favorable en este aspecto (ver gráfico 3). 


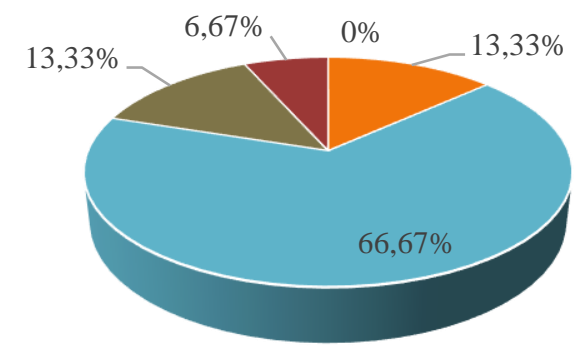

- Excelente - Muybien - Bien - Regular - Malo

Gráfico 3 Opinión hacia la modalidad, con respecto a las actividades académicas por semana

Fuente: Datos de la investigación, 2019

Los resultados obtenidos en las gráficas 1,2 y 3 , coinciden los resultados obtenidos por Madrigal, Edu y Merla (2018), indicando que "entre las razones por las que los estudiantes les gusta cursar la modalidad se encuentra el tiempo, ya que permite administrarlo, aprovecharlo, organizarlo mejor y ahorrarlo" (p. 564), también en Mortis, et al. (2015), los estudiantes mencionan que "la administración del tiempo se relaciona de manera directa con "el tiempo y la disponibilidad" (...). Esto es así, porque tomar un curso de esta modalidad se considera un "ahorro de tiempo" (...), ya que tiene "la ventaja de administrarte con las tareas" (p. 84).

Esta modalidad fue construida e implementada para aquellos egresados que se encontraban en el campo laboral, brindándoles una oportunidad de estudios para continuar con su preparación profesional de nivel licenciatura, las opiniones en este rubro fueron muy favorables, debido a que se encuentran empleados, a que no se presentan todos los días a clases y que el horario de estudios es flexible (ver gráficos 4,5 y 6 ).

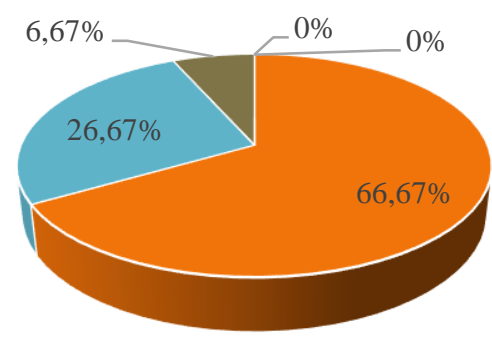

- Excelente - Muy bien - Bien - Regular - Malo

Gráfico 4 Opinión hacia la modalidad como una oportunidad de estudios, debido a su empleabilidad Fuente: Datos de la investigación, 2019

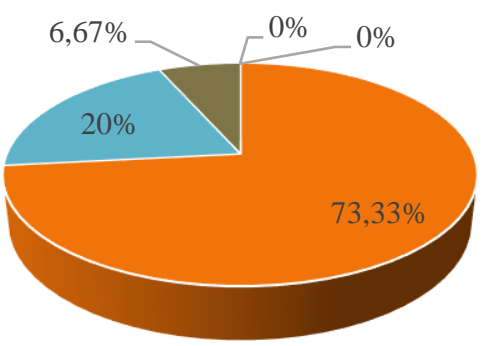

- Excelente - Muybien - Bien - Regular - Malo

Gráfico 5 Opinión hacia la modalidad como una oportunidad de estudios, debido a que no se presentan todos los días a la UT

Fuente: Datos de la investigación, 2019

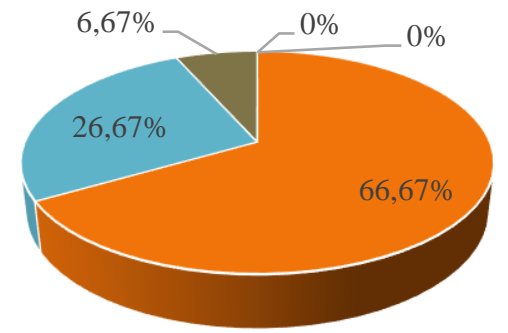

- Excelente - Muy bien - Bien - Regular - Malo

Gráfico 6 Opinión hacia la modalidad como una oportunidad de estudios, debido a que no existe horario de clases

Fuente: Datos de la investigación, 2019

Los resultados obtenidos en las gráficas 5 y 6, coinciden con los resultados de Mortis, et al. (2015), donde manifiesta que "los alumnos consideran que un aspecto relevante de la administración del tiempo es la "flexibilidad" (...), ya que brinda la "oportunidad de avanzar en las actividades" (...) y "es una oportunidad" (...) para ahorrar tiempo, porque "no hay horario fijo" (...), "se adecúa a su ritmo de trabajo" (p. 85), además, Pérez y Saker (2013), mencionan que "los estudiantes muestran estar en completo acuerdo con que en la plataforma virtual se puede trabajar al ritmo de cada estudiante y en un horario flexible" (p. 162)

Estudiar esta modalidad para nuestros estudiantes, fue muy favorable de acuerdo a su opinión, debido a que mencionan haber mejorado su desempeño profesional en el lugar donde laboran, además que les representa una oportunidad de mejorar sus ingresos económicos (ver gráficos 7 y 8 ). 


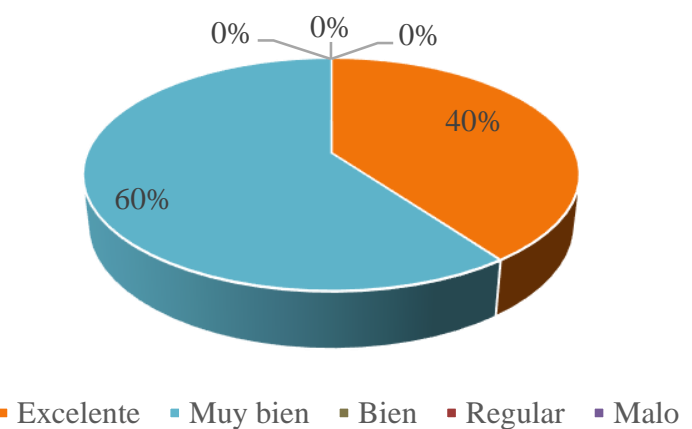

Gráfico 7 Opinión hacia la modalidad, con respecto al beneficio profesional obtenido

Fuente: Datos de la investigación, 2019

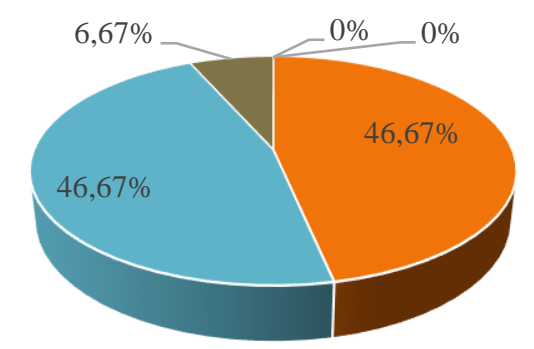

- Excelente - Muy bien - Bien - Regular - Malo

Gráfico 8 Opinión hacia la modalidad, con respecto al mejoramiento en su nivel de ingresos

Fuente: Datos de la investigación, 2019

La definición de lineamientos y el apegarse a ellos es de suma importancia; por lo que los estudiantes sugieren que:

"Se deben establecer políticas y
reglamentos en cuanto los tiempos de
publicación y cantidad de actividades que
asignan los docentes, ya que algunos utilizan
criterios distintos." (OpE1).

"Es importante establecer politicas y que se respeten en las que se den a conocer a todos los alumnos y docentes, los tiempos de entrega de actividades, fechas de asignación de actividades, cantidad de actividades asignadas por materia, sesiones online; ya que no se tuvo un criterio bien establecido, algunos docentes asignaban más actividades que otros y publican su material y actividades hasta cuatro días después de la fecha de inicio de semana" (OpE2).

Las actividades que se desarrollan en las materias deben tener una planeación, de tal manera que no se pierda el enfoque pedagógico del proceso de enseñanza - aprendizaje, por lo que, los estudiantes sugieren que:
"Debe existir un seguimiento por parte de la Dirección a las actividades de la modalidad mixta y sobre todo que se realice una adecuada planeación, debido a que en tres cuatrimestres existieron muchas inconsistencias en cuanto a la planeación de actividades (OpE4).

\section{Agradecimientos}

El presente estudio fue financiado con recursos propios de la Universidad Tecnológica de la Selva, a través de la Convocatoria Fomento a la Formación de Recursos Humanos de Alta Calidad y Desarrollo de Proyectos de Investigación. Por lo anterior, se extiende un agradecimiento a la misma por el apoyo brindado.

Se hace un agradecimiento particular a la Lic. Guiomar Cameras Martínez, por las revisiones y correcciones realizadas a la traducción del resumen del presente artículo.

\section{Conclusiones}

Los resultados obtenidos sobre la plataforma educativa, demuestran que existe una mayoría de estudiantes que esta conforme con el aspecto y uso de ella, sin embargo, existen algunos aspectos que se deben mejorar, con el fin de estandarizar la presentación de los recursos didácticos y su facilidad de localización.

En relación a los docentes, los estudiantes opinan que los recursos didácticos proporcionados fueron los adecuados para su formación profesional, demostraron profesionalismo en la atención oportuna de las dudas y envío de las retroalimentaciones de sus actividades y que cuentan con un amplio dominio de las asignaturas impartidas, por lo que se concluye que los docentes tuvieron muy buen desempeño al trabajar en esta modalidad.

Respecto a la modalidad de estudios, los estudiantes opinan que es excelente para continuar con sus estudios profesionales, ya que en cuanto al tiempo de estudios pueden administrarlo como ellos consideren, debido a que mezclan sus estudios con sus responsabilidades laborales, además esperan mejorar su desempeño profesional en sus actividades laborales, así como, mejorar sus condiciones económicas al conseguir un título profesional de nivel licenciatura. 
Las perspectivas de los estudiantes que se encuentran inmersos en el campo laboral con relación a la modalidad mixta, han demostrado que se debe continuar con la oferta de esta modalidad de estudios, ya que les representa una oportunidad de continuar con su formación profesional de nivel licenciatura dentro del Subsistema de las Universidades Tecnológicas.

\section{Líneas abiertas de investigación}

Es preciso señalar que existen aspectos dentro del proyecto de investigación que no se tomaron en cuenta y que dan pauta a futuras investigaciones, siendo éstas, la calidad de los recursos didácticos, la perspectiva de los docentes hacia esta modalidad, competencias digitales de los docentes, la interacción alumno - maestro, que permitirá consolidar esta modalidad en la institución.

\section{Referencias}

Almarza Franco, Y., y Pirela Morillo, J. (2010). Las bibliotecas universitarias y el enfoque blearning. Biblioteca Universitaria, 13 (2), 13. Disponible en: http://revistas.unam.mx/index.php/rbu/article/vi ew/24110/22642

Alonso Esquivel, A. M. (2010). Evaluación de la satisfacción del alumnado de cursos virtuales en la Empresa de Telecomunicaciones de Cuba, S.A. (ETECSA). Edutec. Revista Electrónica De Tecnología Educativa, (32), a134. https://doi.org/10.21556/edutec.2010.32.439

Asociación de Internet MX (2017). Educación en Línea en México 2017. Disponible en: http://mhapps01.cloudapp.net/amipci/images/Es tudioEducacionenLinea2017.pdf

Asociación de Internet MX (2018). Educación en Línea en México 2018. Disponible en: https://www.asociaciondeinternet.mx/es/compo nent/remository/Educacion-en-Linea-enMexico/Estudio-de-Educacion-en-LineaAIMX-2019/lang,es-es/?Itemid=

Chávez, M. A. (2015). Cómo enseñar a las nuevas generaciones digitales. Revista Electrónica de Investigación Educativa, 17 (2), 1-4. Disponible en: http://redie.uabc.mx/vol17no2/contenidocanor.html
Coaten, N. (2003). Blended e-learning. Educaweb $69 . \quad$ Disponible en: http://www.educaweb.com/esp/servicios/monog rafico/formacionvirtual/1181076.asp

Contreras Bravo, L. E., González Guerrero, K., y Fuentes López, H. J. (2011). Uso de las TIC y especialmente del blended learning en la enseñanza universitaria. Revista Educación y Desarrollo Social, 5 (1), 151-160.

E. Marsh II, G., C. McFadden, A. y Jo, B. (2003). Blended Instruction: Adapting Conventional Instruction for Large Classes. Online Journal of Distance Learning Administration. VI (IV).

Fernández Aedo, R., y Panadeiro, A. (2009). Influencias de las Tecnologías de la Información y las Comunicaciones en la Universalización de la Enseñanza. RIED. Revista Iberoamericana de Educación a Distancia, 12 (1), 63-75.

Fernández Morales, K. y Vallejo, A. (2014). La educación en línea: una perspectiva basada en la experiencia de los países. Revista de Educación y Desarrollo. 29. 29-39.

Hernández Sampieri, R., Fernández Collado, C., y Baptista Lucio, M. (2014). Metodología de la investigación (Sexta edición). México: McGraw-Hill.

López-Roldán, P.; Fachelli, S. (2015). La encuesta. En P. López-Roldán y S. Fachelli, Metodología de la Investigación Social Cuantitativa. Bellaterra (Cerdanyola del Vallès): Dipòsit Digital de Documents, Universitat Autònoma de Barcelona. Capítulo II.3. Edición digital: http://ddd.uab.cat/record/163567

Madrigal Lozano, M. G., Edu Dörfer, C. y Merla González, A. E. (2018). La modalidad mixta desde la perspectiva de los estudiantes. Disponible en: https://www.researchgate.net/ publication/329758347_La_modalidad_mixta_ desde_la_perspectiva_de_los_estudiantes

Montemayor Flores, B. (2015). El aula virtual como complemento de clase presencial. Reporte de experiencia. México, D.F.

Mortis Lozoya, S., Del Hierro Parra, E., García López, R., y Manig Valenzuela, A. (2015). La modalidad mixta: un estudio sobre los significados de los estudiantes universitarios. Innovación Educativa, 15 (68), 73-97.

DOMINGUEZ-GUTU, Jesús, GORDILLO-ESPINOZA, Emmanuel y CONSTANTINO-GONZÁLEZ, Fernando Exiquio. La modalidad mixta una alternativa de continuidad de estudios para egresados inmersos en el campo laboral. Revista de Educación Superior. 2019 
Pérez Cervantes, M. L. y Saker, A. F. (2013). Importancia del uso de las plataformas virtuales en la formación superior para favorecer el cambio de actitud hacia las TIC; Estudio de caso: Universidad del Magdalena, Colombia. Revista Iberoamericana de Evaluación Educativa, 6 (1). 153-166.

Sierra Varón, C. A. (2011). La educación virtual como favorecedora del aprendizaje autónomo. Panorama, 5 (9), 75 - 87.

Universidad Tecnológica de la Selva (2013). Programa Institucional Universidad Tecnológica de la Selva 2013-2018. Disponible en: http://www.utselva.edu.mx/MarcoLegal/Marco Legal/ProgramaInstitucion1/Documento-PlanUTSelva-Actual.pdf

Vera, F. (2008). La modalidad blended-learning en la educación superior. Disponible en:

$\mathrm{http}: / / \mathrm{www} \cdot u$ temvirtual.cl/nodoeducativo/wpcontent/uploads/2009/03/fvera_2.pdf

Zubieta García, J., y Rama Vitale, C. (2015). La educacion a distancia. México, D. F.: Universidad Nacional Autónoma de México. 